Pesq. Vet. Bras. 31(2):104-110, fevereiro 2011

\title{
Prevalência e etiologia da mastite bovina na bacia leiteira de Rondon do Pará, estado do Pará1
}

\author{
Carlos Magno C. Oliveira ${ }^{2^{*}}$, Melina Garcia S. Sousa ${ }^{2}$, Natália da Silva e Silva ${ }^{2}$, \\ Carla L. Mendonça ${ }^{3}$, José Alcides S. Silveira ${ }^{2}$, Ricardo Pedroso Oaigen², \\ Stefano Juliano T. Andrade ${ }^{2}$ e José Diomedes Barbosa ${ }^{2}$
}

\begin{abstract}
Oliveira C.M.C., Sousa M.G.S., Silva N.S., Mendonça C.L., Silveira J.A.S., Oaigen R.P., Andrade S.J.T. \& Barbosa J.D. 2011. [Prevalence and etiology of bovine mastitis in the dairy region of Rondon do Pará, state of Pará.] Prevalência e etiologia da mastite bovina em rebanhos leiteiros na região de Rondon do Pará, estado do Pará. Pesquisa Veterinária Brasileira 31(2):104-110. Central de Diagnóstico Veterinário, Faculdade de Medicina Veterinária, Campus Castanhal, Universidade Federal do Pará, Rua Maximino Porpino da Silva 1000, Castanhal, PA 68740-080, Brazil. E-mail: cmagno@ufpa.br

The prevalence and the etiology of bovine mastitis in the dairy region of the county of Rondon of Pará were investigated. The sensibility and the resistance of the isolated agents to the antimicrobiotics was evaluetad; 237 mixed-bred milk cows were used on nine properties, which were hand milked once a day and kept on Brachiaria brizantha pasture, with supply of mineral salt and water ad libitum. Clinical examination of the mammary gland, the test of the sieve and California Mastitis Test were performed. Of the 935 examined mammary quarters, $6.6 \%$ had subclínical mastitis, $1.3 \%$ clinical mastitis, and $92.1 \%$ were negative. The isolated bacteria in the clinical mastitis were coagulase negative Staphylococcus spp. (25\%), Staphylococcus aureus (16.7\%), Streptococcus spp. (8,3\%), and Corynebacterium spp. (8.3\%). In the subclínical mastitis coagulase negative Staphylococcus spp. (32.3\%), Staphylococcus aureus (17.7\%), Staphylococcus intermedius (1.6\%), Streptococcus spp. (4.8\%), Corynebacterium spp. (4.8\%), and coagulase negative Staphylococcus spp./S.aureus (1.6\%) were isolated. There was no microbial growth in $41.7 \%$ of the samples with clinical mastitis and in $37.1 \%$ with subclínical mastitis. In the antibiogram, $100 \%$ of the isolated negative coagulase Staphylococcus spp., S. aureus, S. intermedius, and Streptococcus spp. were sensitive to sulfazotrim. On the other hand, Corynebacterium spp. were $100 \%$ resistant to this same antimicrobiotic. Cefalotina, cefoxitina and gentamicina were efficient against the isolated Staphylococcus spp. which represent the greater part of the mastitis agents in this study. Mastitis was diagnosed in all flocks studied, however the number of affected animals was considered low; this probably is due to low milk production of the cows and to permanence of the calf with the mother after milking, what favors emptying the mammary gland. But hygienic sanitary measures and management practices have to be improved.
\end{abstract}

INDEX TERMS: Bovine mastitis, etiology, Staphylococcus spp., antibiogram, Pará.

\footnotetext{
${ }^{1}$ Recebido em 27de abril de 2010.

Aceito para publicação em 14 de setembro de 2010.

${ }^{2}$ Central de Diagnóstico Veterinário (Cedivet), Faculdade de Medicina Veterinária, Campus Castanhal, Universidade Federal do Pará (UFPA), Rua Maximino Porpino da Silva 1000, Castanhal, PA 68740080, Brasil. "Autor para correspondência: cmagno@ufpa.br

${ }^{3}$ Clínica de Bovinos, Campus Garanhuns, Universidade Federal Rural de Pernambuco (UFRPE), Av. Bom Pastor s/n, Cx. Postal 152, Garanhuns, PE 55292-901, Brasil.
}

RESUMO.- O objetivo do presente trabalho foi pesquisar a prevalência e a etiologia da mastite bovina na bacia leiteira do município de Rondon do Pará, bem como avaliar o perfil de sensibilidade e resistência dos agentes isolados frente aos antimicrobianos. Foram avaliadas 237 vacas mestiças de aptidão leiteira, pertencentes a nove propriedades, as quais utilizavam ordenha manual uma vez ao dia e sistema de criação extensivo em pastagens de Brachiaria brizantha, 
com fornecimento de sal mineral e água ad libitum. Realizou-se o exame clínico da glândula mamária, o teste da caneca telada e o California Mastitis Test. Dos 935 quartos mamários avaliados, 6,6\% apresentaram mastite subclínica, 1,3\% mastite clínica e 92,1\% foram negativos. As bactérias isoladas na mastite clínica foram Staphylococcus spp. coagulase negativo (25\%), Staphylococcus aureus $(16,7 \%)$, Streptococcus spp. (8,3\%) e Corynebacterium spp. $(8,3 \%)$. Na mastite subclínica foram Staphylococcus spp. coagulase negativo (32,3\%), Staphylococcus aureus $(17,7 \%)$, Staphylococcus intermedius (1,6\%), Streptococcus spp. $(4,8 \%)$, Corynebacterium spp. $(4,8 \%)$ e Staphylococcus spp. coagulase negativo/S. aureus (1,6\%). Não houve crescimento microbiano em $41,7 \%$ das amostras com mastite clínica e $37,1 \%$ com mastite subclínica. No antibiograma, $100 \%$ dos isolados de Staphylococcus spp. coagulase negativo, $S$. aureus, S. intermedius, e Streptococcus spp. foram sensíveis ao sulfazotrim. Por outro lado Corynebacterium spp. foi $100 \%$ resistente ao mesmo antimicrobiano. A cefalotina, cefoxitina e gentamicina, apresentaram eficácia frente às bactérias isoladas do gênero Staphylococcus spp., as quais neste trabalho representam a grande maioria dos agentes causadores de mastite. A mastite foi diagnosticada em todos os rebanhos pesquisados, contudo o número de animais acometidos foi considerado baixo; isso provavelmente deve-se à baixa produção de leite dos animais e a permanência do bezerro ao pé após a ordenha, o que favorece o esvaziamento da glândula mamária. Diante disso, faz-se necessário que medidas higiênico-sanitárias e de manejo sejam adotadas.

TERMOS DE INDEXAÇÃO: Mastite bovina, etiologia, Staphylococcus spp., antibiograma, Pará.

\section{INTRODUÇÃO}

No estado do Pará, a mesorregião do Sudeste Paraense é composta por 39 municípios que se destacam por produzir $79 \%$ da produção de leite do estado (IBGE 2007). Nessa mesorregião a produção de leite transformou-se numa importante atividade econômica, constituindo-se hoje no segmento comercial mais expressivo para a agricultura familiar. Dentro dessa mesorregião, destaca-se o município de Rondon do Pará que produz em torno 130 mil litros de leite por dia, representando 9,3\% da produção do Sudeste Paraense. Apesar da expressividade na produção de leite dentro do estado do Pará, não existe estudo sobre a prevalência da mastite bovina nos rebanhos leiteiros dessa mesorregião.

A infecção da glândula mamária é responsável por grandes prejuízos econômicos, por causar redução na produção e na qualidade do leite dos quartos mamários afetados, além de interferir no processo industrial de laticínios (Hillerton 1996). A perda de produção pode ser drástica, especialmente quando se infectam uma porcentagem significativa do rebanho (Rebhun 2000). Podendo reduzir em até 50\% a produção leiteira, diminui a vida produtiva da vaca, havendo perda de 15\% de leite por vaca (Ladeira 2007).
A mastite caracteriza-se por uma inflamação da glândula mamária, responsável por alterações físicas, químicas e bacteriológicas no leite e/ou alterações no tecido glandular (Radostits et al. 2002). Podendo ser classificada em clínica ou subclínica (Philpot \& Nickerson 1991). A forma clínica da enfermidade apresenta sinais visíveis; enquanto que, a forma subclínica apresenta aumento na contagem de células somáticas (CCS) do leite, podendo ser diagnosticada através de métodos como California Mastitis Test (CMT) (Grunert 1993).

Radostits et al. (2002), classificam os agentes etiológicos da mastite bovina em patógenos contagiosos (Staphylococcus aureus, Streptococcus agalactiae, Corynebacterium bovis e Mycoplasma bovis), patógenos ambientais (Streptococcus uberis, Streptococcus dysgalactiae, Streptococcus equinus, Escherichia coli, Klebsiella spp., Citrobacter spp., Enterobacter spp. e Pseudomonas spp.), patógenos secundários ou menores (Staphylococcus spp. coagulasenegativas) e patógenos incomuns (Arcanobacterium pyogenes, Nocardia spp., Pasteurella spp., Mycobacterium bovis, Bacillus cereus, Serratia marcescens, algumas espécies de bactérias anaeróbias, fungos e leveduras).

O objetivo deste trabalho é pesquisar a prevalência e a etiologia da mastite bovina na bacia leiteira do município de Rondon do Pará, bem como avaliar o perfil de sensibilidade e resistência dos agentes isolados frente aos antimicrobianos testados.

\section{MATERIAL E MÉTODOS}

Foram estudadas 237 vacas mestiças de aptidão leiteira, em diferentes fases de lactação, pertencentes a nove propriedades localizadas na bacia leiteira de Rondon do Pará, estado do Pará. As propriedades utilizavam ordenha manual uma vez ao dia e sistema de criação extensivo em pastagens de Brachiaria brizantha, com fornecimento de sal mineral e água ad libitum. Aplicou-se um questionário por propriedade para identificar os fatores associados à ocorrência da infecção da glândula mamária.

Inicialmente realizou-se o exame clínico da glândula mamária e em seguida, o teste da caneca de fundo escuro ou telada (Fig.1) de acordo com Grunert (1993); quartos mamários sem mastite clínica foram submetidos ao CMT (Fig.2) segundo Schalm \& Noorlander (1957). Os animais em fase colostral e aqueles que estavam no final da lactação não foram submetidos aos testes.

Foram colhidas individualmente amostras de leite $(4 \mathrm{ml})$ dos quartos mamários positivos aos testes da caneca telada e CMT, após a assepsia dos tetos, em tubos de ensaio esterilizados, devidamente identificados, acondicionados em caixas isotérmicas com gelo e remetidas sob refrigeração ao Laboratório Clínico da Clínica de Bovinos, Campus Garanhuns, da Universidade Federal Rural de Pernambuco (UFRPE).

As amostras de leite foram semeadas em placas de Petri contendo Ágar sangue desfibrinado de ovino a 5\% e Ágar MacConkey e incubadas a $37^{\circ} \mathrm{C}$. As leituras foram realizadas às 24 , 48,72 e 96 horas, sendo observadas as características culturais das colônias (morfologia, produção de pigmento e hemólise) e morfo-tintoriais, por meio da coloração de Gram, que permitiu uma visão preliminar das bactérias envolvidas no processo (Quinn et al. 2004). 


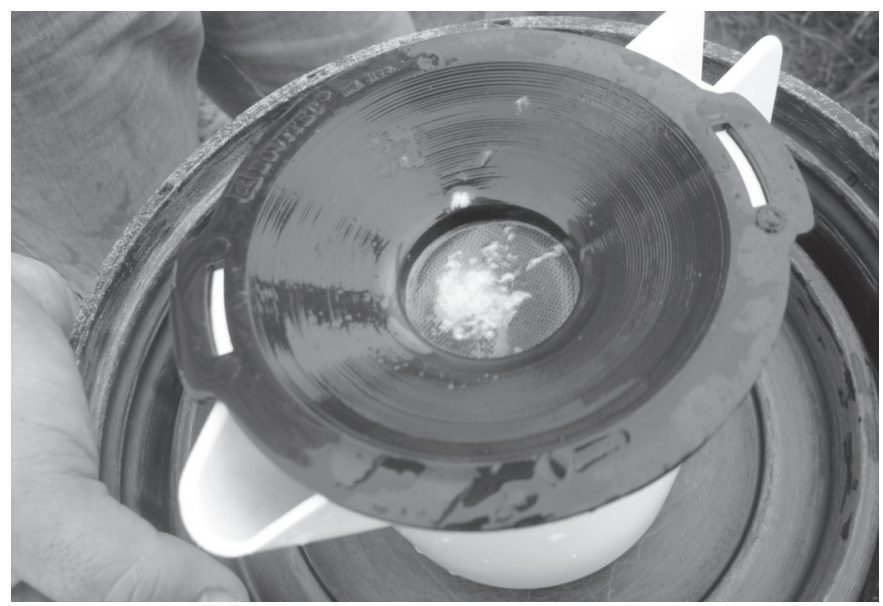

Fig.1. Teste da caneca de fundo escuro ou telada com presença de grumos, Rondon do Pará, PA.

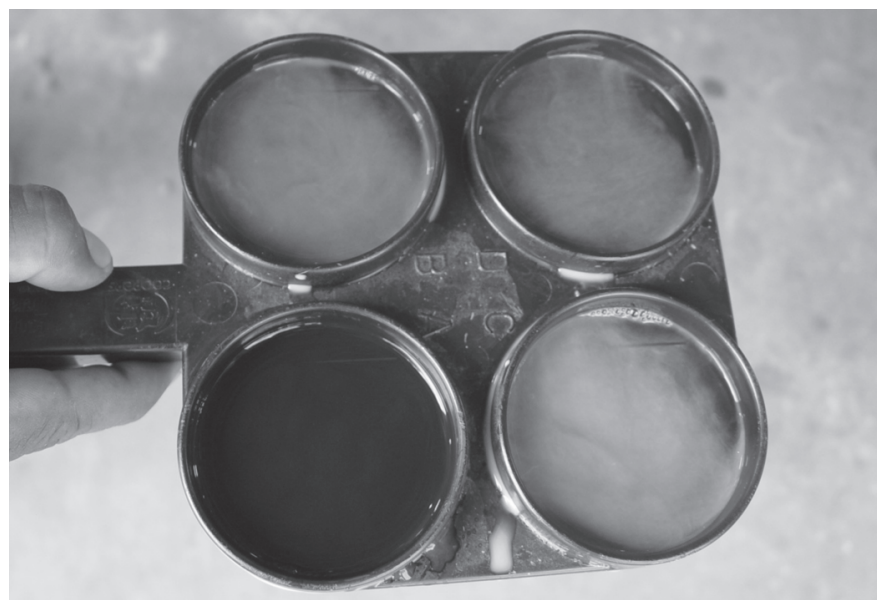

Fig.2. Teste California Mastitis Test, com reação positiva em três amostras de leite de quartos mamários, Rondon do Pará, PA.

O teste de sensibilidade e resistência aos antimicrobianos foi realizado pelo método de difusão por discos (Bauer et al. 1966), seguindo-se as especificações do Clinical and Laboratory Standards Institute (CLSI) (2005). Desta forma, 3 a 5 colônias com as mesmas características morfológicas foram transferidas para tubos de ensaio contendo 4 a $5 \mathrm{ml}$ de caldo Casoy (Caldo Casoy Vetec $^{\circledR}$ ) e incubadas a $37^{\circ} \mathrm{C}$ por 2 a 6 horas, para obter uma turbidez equivalente a 0,5 da escala padrão de McFarland. Em seguida foi realizada a semeadura, com swab estéril sobre a superfície do meio de Ágar Mueller-Hinton (Mueller-Hinton MicroMed ${ }^{\circledR}$ ). A etapa seguinte consistiu na deposição dos discos, fazendo-se leve pressão para permitir o contato entre os mesmos e a superfície do meio inoculado. Utilizaram-se discos (Sensibiodisc/Cecon ${ }^{\circledR}$, Sensifar-Vet/Cefar Diagnóstica $\left.{ }^{\circledR}\right)$ impregnados com os seguintes antimicrobianos: Ampicilina $(10 \mu \mathrm{g})$, Cefalotina $(30 \mu \mathrm{g})$, Cefoxitina $(30 \mu \mathrm{g})$, Enrofloxacina $(5 \mu \mathrm{g})$, Eritromicina $(15 \mu \mathrm{g})$, Estreptomicina $(10 \mu \mathrm{g})$, Gentamicina $(10 \mu \mathrm{g})$, Kanamicina $(30 \mu \mathrm{g})$, Neomicina $(30 \mu \mathrm{g})$, Oxacilina $(1 \mu \mathrm{g})$, Penicilina G (10 UI), Sulfazotrim $(25 \mu \mathrm{g})$ e Tetraciclina $(30 \mu \mathrm{g})$. Para o controle na qualidade de execução e confiabilidade dos resultados obtidos, cepas padrão de Escherichia coli ATCC 25922, Staphylococcus aureus ATCC 25923, Pseudomonas aeruginosa ATCC 27853 e Enterococ- cus faecalis ATCC 29212, foram testadas sob as mesmas condições de meios de cultivo e incubação. A incubação ocorreu à temperatura de $37^{\circ} \mathrm{C}$ por um período de 24 horas, quando então foi realizada a leitura de acordo com o diâmetro de sensibilidade apresentado por cada amostra frente aos diversos antimicrobianos utilizados.

Os dados obtidos foram submetidos à estatística descritiva, sendo calculado o número de casos de mastite clínica e subclínica, os microrganismos isolados e o perfil de sensibilidade frente aos antimicrobianos.

\section{RESULTADOS E DISCUSSÃO}

Dos 237 animais avaliados, 4,6\% (11/237) das vacas apresentaram mastite clínica, 15,6\% (37/237) apresentaram mastite subclínica e 79,7\% (189/237) estavam sadias. Analisando-se o percentual de quartos mamários afetados, foram examinados 948 quartos e 13 correspondiam a quartos mamários sem produção de leite. Portanto, foram submetidos aos testes 935 quartos em produção, destes 6,6\% (62/935) apresentaram mastite subclínica, 1,3\% (12/935) mastite clínica e 92,1\% (861/935) estavam sadios.

A prevalência da mastite subclínica foi relativamente baixa quando comparada a outros autores. Beloti et al. (1997) obtiveram prevalência de $14,66 \%$ de mastite subclínica no Norte do Paraná, trabalhando com animais da raça Holandesa. Ribeiro et al. (2003) e Ribeiro et al. (2006), encontraram prevalência de $37,7 \%$ e $31,17 \%$ respectivamente, em unidades produtoras de leite em rebanhos das raças Jersey e Holandesa que utilizavam sistema de ordenha mecânica no sul do Rio Grande do Sul. Ferreira et al. (2007), no estado do Piauí, realizaram estudo em oito propriedades rurais que utilizavam ordenha manual e observaram $41,10 \%$ de quartos mamários com mastite subclínica. Martins et al. (2010), obtiveram prevalência de 65,0\% no estado do Mato Grosso, em rebanhos com sistema de ordenha mecânica. Lins \& Marreiros (1992), encontraram $21,94 \%$ de mastite subclínica no estado do Piauí; Costa et al. (1995), 46,54\%; Costa et al. (1996), 48,8\%; Samara et al. (1996), 16,50\% em São Paulo; Almeida \& Silva (1998), $19,80 \%$ em Pernambuco. Esta baixa ocorrência pode estar relacionada à baixa produção leiteira dos animais do presente trabalho, com média de 3,7 L/animal/dia, pois segundo Bramley et al. (1996), em animais de alta produção, há maior incidência de mastite, por ser uma categoria de maior exigência nutricional, apresentando maior susceptibilidade à enfermidade. Barbosa et al. (2009) realizaram um estudo comparativo entre os tipos de ordenha e a CCS, no qual concluíram que a ordenha manual apresentou menores valores de CCS, justificando também a baixa prevalência neste trabalho.

No presente trabalho os bezerros permaneceram com as mães desde a ordenha até as 14 horas do dia, o que contribuiu para a retirada do leite residual na glândula mamária. De acordo com Brandão et al. (2008), a presença do bezerro contribui para uma menor incidência da mastite subclínica, pois há maior eficiência na ejeção do leite em decorrência da maior liberação de oxitocina. Para Das et al. (1999) e Combellas et al. (2003), a redução da mastite 
subclínica em vacas que permanecem com o bezerro ao pé após a ordenha também se deve ao maior esgotamento da glândula mamária após a mamada do bezerro e à ação antimicrobiana da saliva.

O índice de mastite clínica $(1,3 \%)$ no presente estudo está próximo aos encontrados por Ribeiro et al. (2003), que detectaram 1,5\% e Ribeiro et al. (2006) que observaram $1,22 \%$ de quartos mamários afetados, ambos os trabalhos realizados em rebanhos leiteiros na região sul do estado do Rio Grande do Sul. Contudo diferem de Pinheiro et al. (2009), que encontraram incidência de $7 \%$ de mastite clínica em Bambuí, MG, em animais cruzados HolandêsGir e de Martins et al. (2010) que observaram 5,8\% de mastite clínica no estado do Mato Grosso. Segundo Fonseca \& Santos (2000) o nível internacionalmente aceito de mastite clínica é em torno de $1 \%$, valor próximo ao encontrado no presente trabalho.

No Quadro 1 estão apresentados as bactérias isoladas das amostras de leite. O percentual e as bactérias isoladas do leite dos quartos com mastite clínica foram $25 \%$ (3/12 amostras) de Staphylococcus spp. coagulase negativo, 16,7\% (2/12) de Staphylococcus aureus, 8,3\% (1/12) de Streptococcus spp. e 8,3\% (1/12) de Corynebacterium spp.. Na mastite subclínica foram isolados 32,3\% (20/62) de Staphylococcus spp. coagulase negativo, 17,7\% (11/ 62) de Staphylococcus aureus, $1,6 \%(1 / 62)$ de Staphylococcus intermedius, 4,8\% (3/62) de Streptococcus spp., 4,8\% (3/62) de Corynebacterium spp. e 1,6\% (1/62) de Staphylococcus spp. coagulase negativo/S. aureus.

Staphylococcus spp. coagulase negativo são frequentemente isolados de rebanhos leiteiros, geralmente associados a casos subclínicos ou clínicos de intensidade leve. Alguns autores classificam essas bactérias como oportunistas (Philpot \& Nickerson 1991), a ocorrência desse agente como o principal patógeno isolado está de acordo com os relatos de Waage et al. (1999), Freitas et al. (2005) e Cunha et al. (2006) que encontraram Staphylococcus spp. coagulase negativo como o agente etiológico mais comumente isolado nas mastites. Porém, diferem dos resulta-

\begin{tabular}{|c|c|c|c|c|}
\hline Microrganismos & $\begin{array}{c}\text { № } \\
\text { amostras }\end{array}$ & $\begin{array}{l}\mathrm{MC}^{1} \\
(\%)\end{array}$ & $\begin{array}{c}\text { № } \\
\text { amostras }\end{array}$ & $\begin{array}{c}\mathrm{MSC}^{2} \\
(\%)\end{array}$ \\
\hline $\begin{array}{l}\text { Staphylococcus spp. } \\
\text { coagulase negativo }\end{array}$ & 3 & 25,0 & 20 & 32,3 \\
\hline Staphylococcus aureus & 2 & 16,7 & 11 & 17,7 \\
\hline Staphylococcus intermedius & - & - & 1 & 1,6 \\
\hline Streptococcus spp. & 1 & 8,3 & 3 & 4,8 \\
\hline Corynebacterium spp. & 1 & 8,3 & 3 & 4,8 \\
\hline $\begin{array}{l}\text { Staphylococcus spp. } \\
\text { coagulase negativo/ } \\
\text { S. aureus }\end{array}$ & - & - & 1 & 1,6 \\
\hline $\begin{array}{l}\text { Não houve crescimento } \\
\text { microbiano }\end{array}$ & 5 & 41,7 & 23 & 37,1 \\
\hline Total & 12 & 100 & 62 & 100 \\
\hline
\end{tabular}

${ }^{1} \mathrm{MC}=$ mastite clínica. ${ }^{2} \mathrm{MSC}=$ mastite subclínica. dos de Watts (1988), Booth (1995), Bramley et al. (1996) e Brito et al. $(1999,2001)$ que encontraram $S$. aureus com maior prevalência nas infecções da glândula mamária, sendo no presente estudo o segundo patógeno mais isolado, tanto na mastite clínica quanto na subclínica. A maior prevalência de Staphylococcus spp. coagulase negativo como causador da mastite clínica e subclínica neste estudo, pode estar relacionado a falta de higiene nos locais de ordenha e das mãos do ordenhador.

Observou-se, neste estudo, que não houve crescimento microbiano em $41,7 \%$ (5/12) das amostras de leite dos quartos mamários com mastite clínica e 37,1\% (23/62) com mastite subclínica. É descrito na literatura que $15 \%$ a 40\% das amostras de leite de casos de mastite clínica podem dar resultados negativos após cultivo microbiológico, mesmo quando a coleta e os métodos de isolamento são aplicados de maneira correta (Bartelett et al. 1992, Olde Reikerink et al. 2008). O não isolamento do agente da mastite nestes casos pode ser por diversos motivos. Entre esses, citam-se a eliminação espontânea da infecção, a baixa concentração dos patógenos no leite, o padrão de eliminação dos microrganismos, que pode alternar entre números elevados e mais baixos, a localização intracelular de determinados patógenos e a presença de substâncias inibitórias no leite. Os resultados negativos são reduzidos, mas não são eliminados quando se empregam técnicas que procuram aumentar as chances de isolamento.

Em 88,9\% (8/9) das propriedades estudadas os currais e locais de ordenha eram de chão batido, em sua maioria, cercados de arame farpado, os quais durante o período seco (junho a novembro) apresentavam pouca lama, e ocasionalmente retirava-se o esterco para a venda ou adubação. Porém, estes no período chuvoso (dezembro a maio), acumulavam muita lama, fezes e urina (Fig.3). Esse tipo de instalação é o utilizado pela maioria dos produtores de leite na região estudada.

Em 11,1\% (1/9) das propriedades, as instalações eram cobertas e com piso cimentado, realizando-se a limpeza

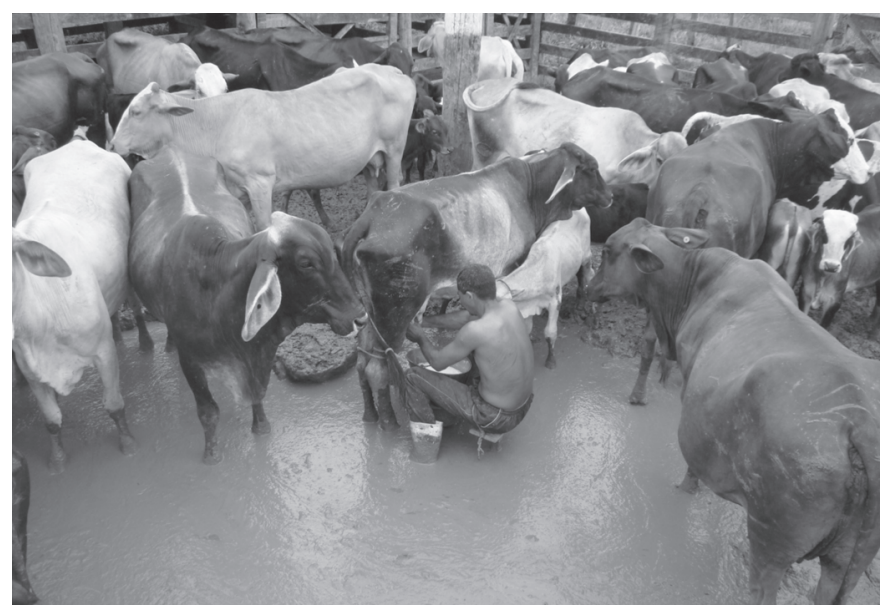

Fig.3. Currais de ordenha durante o período chuvoso com acúmulo de lama e fezes, Rondon do Pará, PA. 
das instalações diariamente, contudo os currais de pré-ordenha eram lamacentos, principalmente no período chuvoso. Nenhuma das propriedades realizava testes para diagnóstico da mastite e não havia higiene por parte do ordenhador no momento da ordenha. Segundo Bramley (1992) e Vianni et al. (1992), fatores como as mãos do ordenhador, as lesões nos tetos e o meio ambiente, expõem as superfícies dos tetos às infecções ocasionando mastite.

Em 33,4\% (3/9) das propriedades havia assistência veterinária, mas somente para o acompanhamento reprodutivo do rebanho. Em 66,7\% (6/9) das propriedades, os animais apresentaram sinais clínicos de deficiência mineral, principalmente de fósforo, como osteofagia e acentuado emagrecimento no pós-parto. Deficiências minerais podem aumentar a susceptibilidade a infecções e em especial à mastite (Müller 2002). A deficiência dietética de Selênio aumenta a susceptibilidade da glândula mamaria a infecções intramamárias (Smith et al. 1984).

De acordo com Philpot \& Nickerson (1991) e Bramley et al. (1996), as práticas de manejo inadequadas e os fatores ambientais contribuem para o desenvolvimento de mastites. Portanto, preconiza-se estabelecer uma linha de ordenha, deixando as vacas infectadas para o final da ordenha (Smith \& Hogan 1992, Peeler et al. 2003), e medidas preventivas como imersão dos tetos pré e pós-ordenha em desinfetante germicida, descarte dos animais com mastite crônica, tratamento de todos os casos clínicos, terapia da vaca seca para todos os animais do rebanho e manutenção dos equipamentos de ordenha (Radostits et al. 2002).

O perfil de sensibilidade, sensibilidade intermediária e resistência dos isolados frente aos antibióticos testados encontra-se no Quadro 2. No antibiograma, $100 \%$ dos isolados de Staphylococcus spp. coagulase negativo foram sensíveis ao sulfazotrim, 91,3\% sensíveis à gentamicina, $87 \%$ sensíveis à cefalotina e cefoxitina, $78,3 \%$ sensíveis à enrofloxacina, neomicina, kanamicina e oxacilina e 73,9\% sensíveis à tetraciclina. A resistência aos antimicrobianos variou de 4,3 a $47,8 \%$.
Dos isolados de Staphylococcus spp. coagulase positivo (S. aureus e $S$. intermedius), $100 \%$ foram sensíveis ao sulfazotrim, 93,3\% sensíveis à tetraciclina e enrofloxacina e $86,7 \%$ sensíveis à neomicina, kanamicina, cefalotina, cefoxitina, gentamicina e oxacilina. A resistência aos antimicrobianos variou de 6,7 a $73,3 \%$.

Os isolados de Streptococcus spp. foram $100 \%$ sensíveis ao sulfazotrim e gentamicina; e $50 \%$ sensíveis aos demais antimicrobianos.

Dos isolados de Corynebacterium spp., $100 \%$ mostraram-se sensíveis à tetraciclina, estreptomicina, neomicina, kanamicina, cefoxitina, cefalotina e gentamicina; e 100\% resistentes à ampicilina, penicilina, oxacilina e sulfazotrim.

De maneira geral, o antimicrobiano com maior eficácia sobre a maioria dos agentes isolados foi o sulfazotrim, resultado semelhante aos encontrados por Bonna et al. (2007), onde Staphylococcus spp. coagulase negativo apresentou $97,5 \%$ de sensibilidade frente ao sulfazotrim, e por Zafalon et al. (2008), com S. aureus apresentando 97,3\% de sensibilidade ao mesmo antimicrobiano, seguido por cefalotina, cefoxitina e gentamicina, apresentando eficácia frente às bactérias isoladas do gênero Staphylococcus spp., as quais neste trabalho representam a grande maioria dos agentes causadores de mastite.

\section{CONCLUSÕES}

Apesar da baixa frequência de casos identificados nesse estudo, a mastite foi diagnosticada em todos os rebanhos pesquisados.

Mesmo com a falta de higiene nas instalações, durante a ordenha e a não utilização de medidas de controle e prevenção, o número de animais acometidos foi considerado baixo; isso provavelmente deve-se à baixa produção de leite dos animais e a permanência do bezerro ao pé após a ordenha, o que favorece o esvaziamento da glândula mamária.

O Sulfazotrim e a gentamicina são os antibióticos de

Quadro 2. Antibiograma dos isolados do leite de vacas com mastite clínica e subclínica, na bacia leiteira de Rondon do Pará

\begin{tabular}{|c|c|c|c|c|c|c|c|c|c|c|c|c|}
\hline \multirow[t]{2}{*}{ Antibióticos } & \multicolumn{3}{|c|}{$\begin{array}{l}\text { Staphylococcus spp. } \\
\text { coagulase negativo (\%) }\end{array}$} & \multicolumn{3}{|c|}{$\begin{array}{l}\text { Staphylococcus spp. } \\
\text { coagulase positivo (\%) }\end{array}$} & \multicolumn{3}{|c|}{$\begin{array}{c}\text { Streptococcus spp. } \\
(\%)\end{array}$} & \multicolumn{3}{|c|}{$\begin{array}{c}\text { Corynebacterium spp } \\
(\%)\end{array}$} \\
\hline & $s^{a}$ & $\mathrm{I}^{\mathrm{b}}$ & $\mathrm{R}^{\mathrm{C}}$ & $\mathrm{S}$ & $\mathrm{I}$ & $\mathrm{R}$ & $\mathrm{S}$ & 1 & $\mathrm{R}$ & $\mathrm{S}$ & 1 & $\mathrm{R}$ \\
\hline Sulfazotrim $(25 \mu \mathrm{g})$ & 100 & 0 & 0 & 100 & 0 & 0 & 100 & 0 & 0 & 0 & 0 & 100 \\
\hline Gentamicina $(10 \mu \mathrm{g})$ & 91,3 & 8,7 & 0 & 86,7 & 13,3 & 0 & 100 & 0 & 0 & 100 & 0 & 0 \\
\hline Cefalotina $(30 \mu \mathrm{g})$ & 87 & 0 & 13 & 86,7 & 0 & 13,3 & 50 & 0 & 50 & 100 & 0 & 0 \\
\hline Cefoxitina $(30 \mu \mathrm{g})$ & 87 & 0 & 13 & 86,7 & 0 & 13,3 & 50 & 0 & 50 & 100 & 0 & 0 \\
\hline Enrofloxacina $(5 \mu \mathrm{g})$ & 78,3 & 4,3 & 17,4 & 93,3 & 0 & 6,7 & 50 & 0 & 50 & 0 & 100 & 0 \\
\hline Neomicina $(30 \mu \mathrm{g})$ & 78,3 & 21,7 & 0 & 86,7 & 13,3 & 0 & 50 & 50 & 0 & 100 & 0 & 0 \\
\hline Kanamicina $(30 \mu \mathrm{g})$ & 78,3 & 17,4 & 4,3 & 86,7 & 13,3 & 0 & 50 & 50 & 0 & 100 & 0 & 0 \\
\hline Oxacilina $(1 \mu \mathrm{q})$ & 78,3 & 0 & 21,7 & 86,7 & 0 & 13,3 & 50 & 0 & 50 & 0 & 0 & 100 \\
\hline Tetraciclina $(30 \mu \mathrm{g})$ & 73,9 & 4,3 & 21,7 & 93,3 & 0 & 6,7 & 50 & 0 & 50 & 100 & 0 & 0 \\
\hline Ampicilina $(10 \mu \mathrm{g})$ & 60,9 & 0 & 39,1 & 40 & 0 & 60 & 50 & 0 & 50 & 0 & 0 & 100 \\
\hline Penicilina G (10 UI) & 60,9 & 0 & 39,1 & 26,7 & 0 & 73,3 & 50 & 0 & 50 & 0 & 0 & 100 \\
\hline Estreptomicina $(10 \mu \mathrm{g})$ & 43,5 & 8,7 & 47,8 & 53,3 & 26,7 & 20 & 50 & 0 & 50 & 100 & 0 & 0 \\
\hline Eritromicina $(15 \mu \mathrm{g})$ & 34,8 & 52,2 & 13,0 & 33,3 & 53,3 & 13,3 & 50 & 0 & 50 & 0 & 100 & 0 \\
\hline
\end{tabular}


escolha para o tratamento da mastite clínica e subclínica nas propriedades estudadas, uma vez que, a maioria das bactérias isoladas foram sensíveis aos mesmos.

A implantação de medidas de diagnóstico e prevenção como o uso da caneca telada, CMT, linha de ordenha e medidas de higiene antes, durante e pós-ordenha poderão contribuir para uma redução no número de casos de mastite nos rebanhos da bacia leiteira de Rondon do Pará e com isso os produtores poderão oferecer às indústrias de laticínios um produto de melhor qualidade.

Agradecimentos.- Ao Programa Institucional de Bolsas de Iniciação Científica PIBIC/Interior/UFPA.

\section{REFERÊNCIAS}

Almeida M.A.C. \& Silva F.F. 1998. Prevalência de mastite subclínica em bovinos por Staphylococcus sp e Streptococcus sp na microrregião de Garanhuns. Ciênc. Vet. Tróp. 1(1):18-24.

Barbosa C.P., Benedetti E. \& Guimarães E.C. 2009. Incidência de mastite em vacas submetidas a diferentes tipos de ordenha em fazendas leiteiras na região do Triângulo Mineiro. Biosci. J. 25(6):121128.

Bartelett P.C., Miller G.Y., Lance S.E. \& Heider L.E. 1992. Clinical mastitis and intramammary infections on Ohio dairy farms. Prev. Vet. Med. 12:59-71.

Bauer A.W. 1996. Antibiotic susceptibility testing by standartized single disk method. Am. J. Clin. Pathol. 45:493-496.

Beloti V., Müller E.E., Freitas J.C. \& Mettifogo E. 1997. Estudo da mastite subclínica em rebanhos leiteiros no norte do Paraná. Seminário Ciências Agrárias, Londrina, 18(1):45-53.

Booth J.M. 1995. Progress in the control of mastitis. Proceedings International Mastitis Seminar, Tel Aviv, International Dairy Federation, Tel Aviv, 3:3-11

Bonna I.C.F., Santos A.P.V., Teixeira G.N. \& Vieira-da-Motta O. 2007. Staphylococcus coagulase-negativos resistentes a drogas isolados de leite de búfalas (Bubalus bubalis). Revta Bras. Ciênc. Vet. 14(2):117-121.

Bramley A.J. 1992. Mastitis, p. 289-300. In: Andrews A.H.R.W., Blowey H. \& Eddy R.G. (Eds), Bovine Medicine, Diseases and Husbandry of Cattle. Blackwell, Oxford.

Bramley A.J., Cullor R.J., Erskine L.K., Fox R.J., Harmon J.S., Hogan S.C., Nickerson S.P., Oliver K.L., Smith L.M. \& Sordillo J.S. 1996. Current Concepts of Bovine Mastitis. National Mastitis Council. $4^{\text {th }}$ ed. Madison, Wisconsin. 64p.

Brandão F.Z., Ruas J.R.M., Silva Filho J.M., Borges L.E., Ferreira J.J., Carvalho B.C., Marcatti Neto A. \& Amaral R. 2008. Influência da presença do bezerro no momento da ordenha sobre o desempenho produtivo e incidência de mastite subclínica em vacas mestiças holandês-zebu e desempenho ponderal dos bezerros. Revta Ceres 55(6):525-531.

Brito M.A.V.P., Brito J.R.F., Ribeiro M.T. \& Veiga V.M.O. 1999. Padrão de infecção intramamária em rebanhos leiteiros: exame de todos os quartos mamários das vacas em lactação. Arq. Bras. Med. Vet. Zootec. 51(2):129-135.

Brito M.A.V.P., Brito J.R.F., Silva M.A.S. \& Carmo R.A. 2001. Concentração mínima inibitória de dez antimicrobianos para amostras de Staphylococcus aureus isoladas de infecção intramamária bovina. Arq. Bras. Med. Vet. Zootec. 53(5):531-537.

Clinical and Laboratory Standards Institute (CLSI/NCCLS) 2005. Performance standards for antimicrobial susceptibility testing: 26. M 100-S15.

Combellas J. \& Tesorero M. 2003. Cow-calf relationship during milking and its effect on milk yield and calf live weight gain. Livest. Res. Rural Develop. 3:1-9.

Costa E.O., Melville P.A., Ribeiro A.R., Watanabe E.T., White C.R. \& Pardo R.B. 1995. Índices de mastite bovina clínica e subclínica nos Estados de São Paulo e Minas Gerais. Revta Bras. Med. Vet. 17(5):78-80.

Costa E.O., Melville P.A., Ribeiro A.R., Watanabe E.T., Carciofi A.C. \& Porada M.S. 1996. Desinfecção mamária pós-ordenha: avaliação de sua eficiência no controle de mastite bovina. Congresso Brasileiro de Medicina Veterinária, Goiânia, GO, p.123.

Cunha A.P., Silva L.B.G., Pinheiro Júnior J.W., Silva D.R., Oliveira A.A., Silva K.P.C. \& Mota R.A. 2006. Perfil de sensibilidade antimicrobiana de agentes contagiosos e ambientais isolados de mastite clínica e subclínica de búfalas. Arqs Inst. Biológico, São Paulo, 73(1):17-21.

Das S.M., Wiktorson H. \& Forsberg M. 1999. Effects of calf management and level of feed supplementation on milk yield and calf growth of zebu and crossbreed cattle in the semi-arid tropics. Livest. Prod. Sci. 59:67-75.

Ferreira J.L., Lins J.L.F.H.A., Cavalcant T.V., Macedo N.A. \& Borjas A.R. 2007. Prevalência e etiologia da mastite bovina no município de Teresina, Piauí. Ciênc. Anim. Bras. 8(2):261-266.

Fonseca L.F.L \& Santos M.V. 2000. Qualidade do leite e controle de mastite. Lemos Editorial, São Paulo. 175p.

Freitas M.F.L., Pinheiro Júnior J.W., Stamford T.L.M., Rabelo S.S.A., Silva D.R., Silveira Filho V.M., Santos F.G.B., Sena M.J. \& Mota R.A. 2005. Perfil de Sensibilidade antimicrobiana in vitro de Staphylococcus coagulase positivos isolados de leite de vacas com mastite no agreste do estado de Pernambuco. Arqs Inst. Biológico, São Paulo, 72(2):171-177.

Grunert E. 1993. Sistema Genital Feminino, p.269-314. In: Dirksen G., Gründer H.D. \& Stöber M. (Eds), Rosenberger: Exame Clínico dos Bovinos. $3^{\text {a }}$ ed. Guanabara Koogan, Rio de Janeiro.

Hillerton J.E. 1996. Controle da mastite bovina. Workshop sobre Programa Integrado da Mastite Bovina, Embrapa-CNPGL, Juiz de Fora, MG, p.6-24.

IBGE 2007. Pesquisa da Pecuária Municipal. Instituto Brasileiro de Geografia e Estatística, Rio de Janeiro.

Ladeira S.R.L. 2007. Mastite bovina, p.359-370. In: Riet-Correa F., Schild A.L., Lemos R.A.A. \& Borges J.R.J. (Eds), Doenças de Ruminantes e Eqüídeos. Vol.1. 3 $3^{\mathrm{a}}$ ed. Editora Pallotti, Santa Maria.

Lins J.L.F.H.A. \& Marreiros V.P.N. 1992. Mamite bovina na bacia leiteira de Teresina, PI. I. Avaliação do sistema de produção. II. Prevalência de mastite. III. Contribuição à análise epidemiológica. Congresso Interna-cional de Medicina Veterinária em Língua Portuguesa, Salvador, BA, p.227-228.

Martins R.P., Silva J.A.G., Nakazato L., Dutra V. \& Almeida Filho E.S. 2010. Prevalência e etiologia infecciosa da mastite bovina na microrregião de Cuiabá, MT. Ciênc. Anim. Bras. 11(1):181-187.

Müller E.E. 2002. Qualidade do leite, células somáticas e prevenção da mastite. II Sul-Leite, Simpósio sobre Sustentabilidade da Pecuária Leiteira na Região Sul do Brasil, Maringá, PR, p.206-217.

Olde Reikerink R.G., Barkema H., Kelton D. \& Scholl D. 2008. Incidence rate of clinical mastitis on Canadian dairy farms. J. Dairy Sci. 91:13661377.

Philpot W.N. \& Nickerson S.C. 1991. Mastitis counter attack: A strategy to combat mastitis. Babson Bros, Naperville. 150p.

Pinheiro M.L.M., Albino F.T., Fonseca E.G., Teixeira R.B. \& Paiva A.L.C. 2009. Avaliação de mastite clinica e subclinica no Setor de Bovinocultura do Instituto Federal de Minas Gerais, Campus Bambuí. II Semana de Ciência e Tecnologia do IFMG Campus Bambuí e II Jornada Científica, Bambuí, MG.

Quinn P.J., Carter M.E., Markey B.K. \& Carter G.R. 1994. Clinical Veterinary Microbiology. Wolfe Publ., London. 330p. 
Radostits O.M., Gay C.C., Blood D.C. \& Hinchcliff K.W. 2002. Clínica Veterinária: um tratado de doenças dos bovinos, ovinos, suínos, caprinos e eqüinos. $9^{a}$ ed. Guanabara Koogan, Rio de Janeiro. 1737p.

Rebhun W.C. 2000. Doenças do Gado Leiteiro. Roca, São Paulo. p.339-377.

Ribeiro M.E.R., Petrini L.A., Aita M.F., Balbinotti M., Stumpf Jr W., Gomes J.F., Schramm R., Martins P.R. \& Barbosa R.S. 2003. Relação entre mastite clínica, subclínica infecciosa e não infecciosa em unidades de produção leiteira na região sul do Rio Grande do Sul. Revta Bras. Agrociênc. 9(3):287-290.

Ribeiro M.E.R., Petrini L.A., Barbosa R.S., Zanela M.B., Gomes J.F., Stumpf Jr W. \& Schramm R. 2006. Ocorrência de mastite causada por Nocardia spp. em rebanhos de unidades de produção leiteira no sul do Rio Grande do Sul. Revta Bras. Agrociênc. 12(4):471-473.

Samara S.I., Prata L.F. \& Dutra I.S. 1996. Diagnóstico da situação sanitária do gado leiteiro em Pitangueiras, SP. III. Mastite. Ars Vet. 12(2):141-147.

Schalm O.W. \& Noorlander D.D. 1957. Experiments and observations leading to development of the California Mastitis Test. J. Am. Vet. Med. Assoc. 130:199-204.
Smith L.K., Harrison J.H., Hancock D.D., Todhunter D.A. \& Conrad H.R. 1984. Effect of vitamin $E$ and selenium supplementation on incidence of clinical mastitis and duration of clinical symptoms. J. Dairy Sci. 67:1293-1300.

Smith K.L. \& Hogan J.S. 1992. Controle da mastite além da imersão dos tetos e da terapia de vacas secas. Simpósio Internacional de Alimentação de Ruminantes ESALQ, Piracicaba, SP, p.235-240.

Vianni M.C.E.A., Nader Filho A.R.P. \& Langenegger J. 1992. Freqüência de isolamentos de Staphylococcus coagulase positiva e coagulase negativa na mastite subclínica em bovinos e sua influência na produção láctea. Arqs Uni-v. Fed. Rural Rio de J. 15(2):187-92.

Waage S., Mork T., Roros A., Aasland D., Hunshamar A. \& Odgaard S.A. 1999. Bacteria associated with clinical mastitis in dairy heifers. J. Dairy Sci. 82(4):712-719.

Watts J.I. 1988. Etiological agents of bovine mastitis. Vet. Microbiol. 16:41-66.

Zafalon L.F., Arcaro J.R.P., Nader Filho A., Ferreira L.M., Castelani L. \& Benvenutto F. 2008. Investigação de perfis de resistência aos antimicrobianos em Staphylococcus aureus isolados na ordenha de vacas em lactação. Revta Inst. Adolfo Lutz 67(2):118-125. 